2002s-12

\title{
Observation, Flexibilité et Structures Technologiques des Industries
}

Marcel Boyer, Armel Jacques, Michel Moreaux

Série Scientifique

Scientific Series

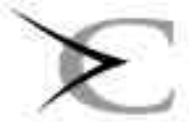

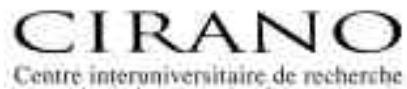

Montréal

Février 2002 


\section{CIRANO}

Le CIRANO est un organisme sans but lucratif constitué en vertu de la Loi des compagnies du Québec. Le financement de son infrastructure et de ses activités de recherche provient des cotisations de ses organisationsmembres, d'une subvention d'infrastructure du ministère de la Recherche, de la Science et de la Technologie, de même que des subventions et mandats obtenus par ses équipes de recherche.

CIRANO is a private non-profit organization incorporated under the Québec Companies Act. Its infrastructure and research activities are funded through fees paid by member organizations, an infrastructure grant from the Ministère de la Recherche, de la Science et de la Technologie, and grants and research mandates obtained by its research teams.

\section{Les organisations-partenaires / The Partner Organizations}

-École des Hautes Études Commerciales

-École Polytechnique de Montréal

-Université Concordia

-Université de Montréal

-Université du Québec à Montréal

-Université Laval

-Université McGill

-Ministère des Finances du Québec

-MRST

-Alcan inc.

- AXA Canada

-Banque du Canada

-Banque Laurentienne du Canada

-Banque Nationale du Canada

- Banque Royale du Canada

- Bell Canada

- Bombardier

- Bourse de Montréal

-Développement des ressources humaines Canada (DRHC)

-Fédération des caisses Desjardins du Québec

-Hydro-Québec

-Industrie Canada

-Pratt \& Whitney Canada Inc.

-Raymond Chabot Grant Thornton

-Ville de Montréal

(C) 2002 Marcel Boyer, Armel Jacques et Michel Moreaux. Tous droits réservés. All rights reserved. Reproduction partielle permise avec citation du document source, incluant la notice (C).

Short sections may be quoted without explicit permission, if full credit, including (C) notice, is given to the source.

Les cahiers de la série scientifique (CS) visent à rendre accessibles des résultats de recherche effectuée au CIRANO afin de susciter échanges et commentaires. Ces cahiers sont écrits dans le style des publications scientifiques. Les idées et les opinions émises sont sous l'unique responsabilité des auteurs et ne représentent pas nécessairement les positions du CIRANO ou de ses partenaires.

This paper presents research carried out at CIRANO and aims at encouraging discussion and comment. The observations and viewpoints expressed are the sole responsibility of the authors. They do not necessarily represent positions of CIRANO or its partners.

\section{ISSN 1198-8177}




\title{
Observation, Flexibilité et Structures Technologiques des Industries*
}

\author{
Marcel Boyer ${ }^{\dagger}$, Armel Jacques et Michel Moreaux $^{\S}$
}

(Version révisée Avril 2001)

\section{Résumé / Abstract}

On étudie l'impact des conditions d'observation sur les choix de flexibilité technologique dans un duopole. Si le marché est de taille importante, la valeur stratégique de la flexibilité est positive et l'observation des choix technologiques favorise l'adoption de technologies flexibles alors que si le marché est de taille plus faible, la valeur stratégique de la flexibilité est négative et l'observation amène les firmes à choisir des technologies moins flexibles.

We study the links between observability and flexible technological choices in a duopolistic market. We show that, in large markets, the strategic value of flexibility is positive and observability of technological choices promotes the adoption of more flexible technologies whereas in small markets, the strategic value of flexibility is negative and observability decreases the level of flexibility chosen by firms.

Mots-clés : Flexibilité, observation, duopole

Keywords: Flexibility, Observation, Duopoly

JEL Classification: D43. L13

\footnotetext{
* Correspondant: Marcel Boyer, CIRANO, 2020 University, 25 ème étage, Montréal, Québec, H3A 2A5, CANADA, email : marcel.boyer@cirano.qc.ca

† Université de Montréal et CIRANO, 2020 rue University \#2500, Montréal, Canada, H3A 2A5.

* CIRANO, 2020 rue University, Montréal, Canada, H3A 2A5.

$\S$ Institut Universitaire de France, Université Toulouse 1, LEERNA et IDEI. Pôle Manufacture, 21 Allée de Brienne, 31042, Toulouse, France.
} 


\section{Introduction}

Les causes du succès ou de l'échec d'une entreprise sont nombreuses et parfois difficiles à cerner. Un argument souvent avancé est que, dans les économies actuelles où la concurrence internationale est de plus en plus forte (au moins dans les secteurs non protégés) et l'environnement économique de plus en plus incertain, une plus grande flexibilité représente un avantage important pour une firme. Cette recherche de flexibilité conduit les firmes à adopter de nouvelles formes d'organisation interne (Milgrom et Roberts, 1990) et à modifier profondément leurs stratégies industrielles (Sanchez, 1995).

La flexibilité ne présente cependant pas que des avantages. Sa mise en place peut être coûteuse : les machines automatisées flexibles sont plus onéreuses que les machines dédiées et l'utilisation optimale d'outils de production plus complexes demande une formation spécifique des employés. En outre, la flexibilité peut être une source de faiblesse stratégique. En effet, l'économie industrielle a largement montré que les firmes pouvaient avoir intérêt à s'engager de façon irréversible à accomplir certaines actions afin d'influencer le comportement de leurs concurrentes. Dans un contexte oligopolistique où l'environnement économique est incertain, les firmes doivent procéder à un arbitrage entre les avantages stratégiques de l'engagement et les possibilités d'adaptation rapide permises par la flexibilité ${ }^{1}$. Cet impact stratégique de la flexibilité est souvent négligé par les auteurs en sciences de gestion et les décideurs. L'objet de cette étude est de mesurer cet impact stratégique et de montrer son influence sur les choix technologiques dans un oligopole. Pour ce faire, on reconsidère le modèle de choix de flexibilité développé par Boyer et Moreaux (1997). Dans ce modèle, les firmes choisissent une technologie puis se font une concurrence en quantités à la Cournot. Il est possible d'isoler l'effet stratégique de la flexibilité sur les choix technologiques, dans ce modèle, en comparant les équilibres de deux jeux : le premier où les choix technologiques sont observables avant que les firmes décident les quantités produites, le second où ils ne le sont pas. Quand le choix technologique de chacune des firmes est observé par sa rivale (jeu avec engagement - AE, ou en boucle fermée pour reprendre la terminologie traditionnelle de la théorie des jeux ${ }^{2}$ ), ce choix peut crédibiliser un engagement

\footnotetext{
${ }^{1}$ Voir Jacques (2000) pour une synthèse de cette littérature.

${ }^{2}$ Dans la littérature sur les jeux dynamiques, on associe généralement l'engagement au choix d'une stratégie en boucle ouverte. L'idée est que les joueurs qui retiennent une telle stratégie s'engagent à prendre une certaine suite de décisions ou à effectuer une certaine suite de mouvements quel que soit le déroulement du jeu. On
} 
à suivre telle politique particulière de production lors de la seconde phase du jeu. Deux facteurs déterminent alors le choix du niveau technologique de flexibilité d'une firme : il doit d'abord être adapté aux caractéristiques de la demande, sa taille moyenne et sa volatilité, c'est l'aspect efficacité, mais il peut aussi conditionner les comportements de production de la firme ellemême ainsi que celui de sa rivale lors de la seconde phase, c'est le facteur stratégique. Quand les choix technologiques ne sont pas observés (jeu sans observation - SO, ou en boucle ouverte), ce facteur stratégique disparaît. Comme le suggèrent Fudenberg et Tirole (1984), la comparaison des équilibres obtenus sous ces deux hypothèses relatives aux conditions d'observation permet de caractériser la valeur stratégique de la flexibilité.

L'intérêt de la comparaison de ces deux jeux n'est pas seulement méthodologique. En pratique, l'environnement informationnel dans lequel les firmes évoluent peut varier d'une industrie à l'autre. Si, dans certaines industries, il est difficilement concevable d'imaginer qu'une firme puisse ignorer la technologie choisie par ses concurrentes, dans d'autres au contraire cette observation peut être imparfaite, comporter un risque d'erreur ou n'intervenir qu'après un long délai. Or, l'introduction d'un aléa, même de faible ampleur, peut parfois modifier fortement les résultats obtenus.

Bagwell (1995) a montré, dans un modèle proche de celui de Stackelberg, que si l'observation de l'action d'un concurrent peut être entachée d'erreur, même avec une très faible probabilité, l'action en question peut perdre tout son pouvoir d'engagement. La logique sous-tendant ce résultat est assez simple. Dans ce modèle, la firme leader choisit une quantité ; la seconde firme observe un signal, qui est la somme de la quantité produite par la première firme et d'un terme aléatoire, et choisit ensuite le niveau de sa production. Dans un équilibre en stratégies pures, le suiveur connaît le niveau de production du leader. Par conséquent, le suiveur ne tient pas compte du signal reçu car si le signal est différent du niveau de production attendu, cette différence est attribuée au terme aléatoire et le suiveur choisit la meilleure réponse au niveau de production du leader. Le leader a alors une forte incitation à s'écarter de ce niveau de production

trouve dans le chapitre 13 de Fudenberg et Tirole (1991) des exemples de jeux où les stratégies en boucle ouverte correspondent à des engagements en ce sens. Dans le contexte du présent modèle, le terme engagement doit être compris comme engagement à suivre telle ou telle politique de production lors de la seconde phase du jeu, le support de cet engagement étant la technologie adoptée lors de la première phase. Il convient alors d'associer engagement à stratégie en boucle fermée plutôt qu'à stratégie en boucle ouverte puisque cet engagement à suivre telle politique n'a de sens que si les choix techniques sont observés par les firmes. 
pour choisir la meilleure réponse à la quantité produite par le suiveur. Il en résulte que le seul équilibre en stratégies pures est l'équilibre de Cournot. L'introduction d'une possibilité d'erreur dans l'observation de l'action du leader annule totalement le pouvoir d'engagement de cette action. Ce résultat est obtenu dans un modèle de type Stackelberg mais a priori sa logique peut être étendue à d'autres modèles de duopole. Dans ce cas, le bon modèle est celui sans possibilité d'engagement (SO). En modifiant légèrement le modèle de Bagwell, Maggi (1999) nuance fortement les conclusions de ce dernier. Il introduit une deuxième source d'incertitude sur le coût de production du leader. Le signal reçu par le suiveur redevient informatif et la quantité choisie par le suiveur est une fonction du signal reçu. Si l'incertitude sur le coût de production du leader est forte [faible] par rapport à l'incertitude sur la qualité du message, l'équilibre du jeu est proche de l'équilibre de Stackelberg [Cournot].

On est ainsi conduit à penser que, dans les industries où la flexibilité est incorporée dans des actifs physiques dont le temps de construction est long et la durée de vie élevée, le bon modèle de choix technologiques est le modèle avec engagement (par exemple, il paraît inconcevable de dissimuler la construction d'une centrale nucléaire ou d'un barrage hydro-électrique). Dans les industries où la flexibilité est plutôt de type organisationnel et peut être modifiée assez rapidement sans que cette modification puisse être observée par les concurrents (e.g. les entreprises de la "nouvelle économie"), le bon modèle de choix technologiques est le modèle sans observation. La réalité est évidemment souvent plus complexe, les possibilités d'observation ne sont en général ni parfaites ni totalement absentes. Elles peuvent en outre résulter d'efforts consentis par les agents, qui cherchent à les améliorer, ou s'efforcent au contraire de dissimuler certaines de leurs actions. Ces efforts peuvent être de nature non-coopérative (divulgation d'information, espionnage industriel) ou coopérative (création d'une association professionnelle chargée de collecter et de divulguer les informations privées de ses membres). Nous considérons cet aspect du processus concurrentiel dans la section 6 .

Nos principaux résultats sont les suivants. Dans les marchés de taille importante, la valeur stratégique de la flexibilité est positive et donc l'observation des choix technologiques favorise l'adoption de technologies flexibles. Dans les marchés de taille plus faible, la valeur stratégique de la flexibilité est négative et l'observation incite les firmes à choisir des technologies moins flexibles. Lorsque les firmes peuvent influencer la structure d'observation du jeu, alors, dans 
les marchés de taille importante, les entreprises choisissent de ne pas observer leurs décisions technologiques réciproques lorsque ce choix est effectué de manière coopérative mais elles optent pour l'observation lorsque le choix est non coopératif. Dans les marchés de taille restreinte, la structure d'observation qui émerge à l'équilibre dépend des valeurs précises des paramètres.

Le plan de l'étude est le suivant. On procède à une revue des principales contributions sur le lien entre l'observation et les choix technologiques de flexibilité, à la section 2. On expose le modèle à la section 3 , et on procède à la comparaison des équilibres des deux types de jeux dans la section 4 . La section 5 est consacrée à un cas intermédiaire où seule l'une des firmes peut observer le choix technologique de sa rivale. On examine, à la section 6 , comment on peut endogénéiser la structure d'observation. On conclut brièvement à la section 7 .

\section{$2 \quad$ La littérature associée}

Trois études ont procédé à des comparaisons d'équilibres en boucle ouverte et en boucle fermée portant sur d'autres aspects de la flexibilité : Vives (1989), Kim, Röller et Tombak (1994) et Boyer, Jacques et Moreaux (2001b).

Vives (1989), à l'instar de Stigler (1939), postule qu'une technologie flexible est une technologie ayant un coût marginal faible. Il étudie deux modèles : un modèle de réduction du coût de production et un modèle de choix de la structure de l'unité de production. Les jeux comprennent deux phases : au cours de la première, les firmes choisissent simultanément leur niveau de flexibilité et, au cours de la seconde, elles se livrent une concurrence à la Cournot. Vives compare les équilibres en boucle ouverte et en boucle fermée de ces deux jeux. Il montre que, dans ces modèles, la valeur stratégique de la flexibilité est toujours positive. De plus, cette valeur augmente lorsqu'augmente l'incertitude sur le niveau de la demande. Ces deux modèles présentent cependant le défaut de ne pas séparer le choix du niveau de flexibilité du choix du niveau de capacité. La flexibilité est choisie non seulement pour mieux réagir aux variations de la demande mais aussi pour pouvoir produire des volumes importants. La modélisation de Vives ne retient qu'un seul paramètre pour représenter ces deux dimensions d'une technologie. Le modèle de Boyer et Moreaux (1997), en introduisant un second paramètre représentant la capacité d'une usine inflexible, permet de mieux prendre en compte ces deux composantes des techniques de production. 
Kim, Röller et Tombak (1994) et Boyer, Jacques et Moreaux (2001b) s'intéressent à une conception différente de la flexibilité introduite par Röller et Tombak (1990). Dans ces modèles, la technologie flexible permet de produire à partir des mêmes installations une gamme de deux biens différenciés tandis que l'autre technologie, dite dédiée, ne permet de produire que l'un de ces deux biens. Le modèle de Kim, Röller et Tombak (1994) est un modèle en temps continu dans lequel les firmes sont dotées initialement de technologies dédiées et peuvent à tout moment adopter la technologie FMS (flexible manufacturing system), ce changement de technique étant alors irréversible. Ces auteurs étudient deux types d'équilibres. Dans le premier, appelé équilibre "silencieux" (silent equilibrium), les firmes s'engagent au début du jeu sur une date d'adoption de la technologie FMS. Cette date ne peut plus ensuite être modifiée et ne peut donc pas dépendre de l'observation d'un changement de technologie par l'autre firme. Dans le second, appelé équilibre "bruyant" (noisy equilibrium), les firmes peuvent conditionner la date d'adoption de la technologie FMS à l'observation de l'adoption de cette technologie par l'autre firme. Dans le second équilibre, la technologie FMS n'est jamais adoptée. En effet, l'adoption de cette technologie par l'une des firmes serait immédiatement suivie de son adoption par l'autre firme. Or les firmes obtiennent des profits plus élevés lorsqu'elles sont toutes les deux équipées de technologies dédiées que lorsqu'elles sont toutes les deux équipées de technologies FMS, car elles sont chacune en position de monopole sur l'un des produits alors qu'avec des technologies FMS, plus coûteuses, elles sont chacune présentes sur les deux marchés ce qui avive la concurrence. Dans le premier équilibre, les firmes adoptent simultanément la technologie FMS dès que le marché, croissant au cours du temps, est suffisamment grand.

Boyer, Jacques et Moreaux (2001b) étudient un modèle ayant une structure de jeu à deux étapes : au cours de la première, les firmes choisissent simultanément leur technologie ; au cours de la seconde, les deux firmes se livrent une concurrence à la Cournot. Ils comparent les équilibres en boucle fermée et en boucle ouverte de ce modèle. Cette comparaison fait apparaître que la flexibilité de gamme a toujours une valeur stratégique positive. L'observation du choix technologique encourage donc l'adoption de technologies FMS. De plus, l'équilibre du jeu en boucle ouverte peut être une configuration technologique où les firmes choisissent des technologies différentes, alors que dans les équilibres en boucle fermée, les firmes choisissent 
toujours la même technologie ${ }^{3}$.

Il est important de remarquer que les équilibres étudiés, dans ces deux derniers modèles, ne sont pas équivalents. Notamment, l'équilibre silencieux n'est pas un équilibre en boucle ouverte. En effet, dans l'équilibre silencieux, la date d'adoption de la technologie FMS ne peut pas être conditionnée à l'adoption de cette technologie par l'autre firme, mais la fonction de décision de production d'une firme dépend de la technologie utilisée par l'autre firme, alors que ce n'est pas le cas dans un équilibre en boucle ouverte ${ }^{4}$. La comparaison des deux équilibres par Kim, Röller et Tombak permet donc de mettre en évidence l'effet stratégique de l'adoption de la technologie FMS sur la date d'adoption de cette technologie par l'autre firme tandis que la comparaison des équilibres en boucle ouverte et en boucle fermée par Boyer, Jacques et Moreaux permet d’isoler l'effet stratégique de l'adoption de la technologie FMS sur le niveau de production de l'autre firme.

\section{Le modèle}

Le modèle servant de cadre à cette étude est celui développé par Boyer et Moreaux (1997). Il s'agit d'un jeu de duopole en deux étapes dans lequel les firmes choisissent simultanément leur technologie respective avant de se livrer, une fois les choix technologiques observés, une concurrence à la Cournot lors de la seconde étape. Deux technologies sont disponibles, la technologie inflexible $i$ et la technologie flexible $f$. La technologie inflexible ne peut être utilisée qu'à sa capacité $q=x$, à un coût variable total $c x$ tandis que la technologie flexible peut être utilisée pour produire n'importe quelle quantité $q \geq 0$, à un coût variable total $c q$. La différence de coût d'investissement entre les deux technologies est égale à $H$ et le coût fixe de la technologie inflexible est posé égal à zéro pour des raisons de simplicité. La fonction de demande inverse est linéaire, $p=\max \{0, \alpha-\beta Q\}$ où $\alpha$ est une variable aléatoire distribuée sur l'intervalle $[\underline{\alpha}, \bar{\alpha}]$, d'espérance $\mu$ et de variance $V ; \underline{\alpha}$ est suffisamment grand pour permettre aux deux firmes de

\footnotetext{
${ }^{3} \mathrm{Du}$ moins si l'on ne considère que les équilibres en stratégies pures.

${ }^{4} \mathrm{Vu}$ les résultats obtenus par Boyer, Jacques et Moreaux (2001b), les équilibres en boucle ouverte dans ce modèle seraient probablement assez différents des équilibres silencieux. Les dates d'adoption de la technologie FMS seraient plus tardives et certains équilibres en boucle ouverte feraient apparaître des phénomènes de diffusion (les firmes adopteraient la technologie FMS à des dates différentes). La première différence conjecturée tient à la disparition de l'effet stratégique positif de l'adoption de la technologie FMS sur le choix de production de l'autre firme ; la seconde à des problèmes de multiplicité d'équilibres et de coordination.
} 
produire lors de la seconde période ${ }^{5,6}$. Les deux firmes observent la valeur de $\alpha$ avant de décider les quantités à produire, mais après avoir choisi leur technologie.

Soit $\Pi^{1}\left(t, t^{\prime}\right)\left[\Pi^{2}\left(t, t^{\prime}\right)\right]$ l'espérance de profit de la firme 1 [2] disposant de la technologie $t\left[t^{\prime}\right]$ faisant face à un concurrent ayant adopté la technologie $t^{\prime}[t]$. De l'étude de Boyer et Moreaux (1997), on tire :

$$
\begin{aligned}
& \Pi^{1}(f, f)=\Pi^{2}(f, f)=\frac{1}{9 \beta}\left[V+(\mu-c)^{2}\right]-H \\
& \Pi^{1}(i, f)=\Pi^{2}(f, i)=\frac{1}{2} x(\mu-\beta x-c) \\
& \Pi^{1}(f, i)=\Pi^{2}(i, f)=\frac{1}{4 \beta}\left[V+(\mu-\beta x-c)^{2}\right]-H \\
& \Pi^{1}(i, i)=\Pi^{2}(i, i)=x(\mu-2 \beta x-c)
\end{aligned}
$$

\section{La comparaison des jeux avec et sans observation}

On analyse d'abord le jeu avec engagement, puis le jeu sans observation. On compare ensuite les choix technologiques d'équilibre et on en déduit la valeur stratégique de la flexibilité.

\subsection{Analyse du jeu avec engagement}

Après avoir caractérisé les fonctions de meilleure réponse des firmes au choix technologique de leur concurrente, on détermine les équilibres qui en résultent.

La flexibilité est la meilleure réponse à l'inflexibilité, $\Pi^{1}(f, i) \geq \Pi^{1}(i, i)$, si et seulement si :

$$
\frac{1}{4 \beta} V+\frac{9}{4} \beta\left[x-\frac{1}{3 \beta}(\mu-c)\right]^{2} \geq H
$$

Cette meilleure réponse est obtenue en comparant le coût de la flexibilité $H$ et les avantages qu'elle procure. Les avantages de la flexibilité comprennent deux composantes. La flexibilité permet d'adapter la production à la volatilité de la demande (premier terme du membre de gauche) ; son attrait augmente donc avec la variance de la demande. La flexibilité permet aussi d'adapter le niveau de production à la taille espérée du marché. En effet, la capacité de la technologie inflexible est exogène, ce qui impose une contrainte forte sur les firmes même en

\footnotetext{
${ }^{5}$ Cela implique que $x \leq \frac{1}{2 \beta}(\underline{\alpha}-c)$.

${ }^{6}$ Dans une étude consacrée aux interactions entre la structure financière des firmes et leur choix technologique de flexibilité, cette hypothèse est levée (Boyer, Jacques et Moreaux, 2001a).
} 
l'absence de toute incertitude sur le niveau de la demande. Le second terme du membre de gauche est le gain permis par la suppression de cette contrainte. Ce gain est d'autant plus grand que la contrainte est forte, et cette dernière est forte lorsque la capacité $x$ est éloignée du niveau de production que choisiraient des firmes se concurrençant en quantités (à la Cournot) sans contrainte sur les quantités.

La flexibilité est la meilleure réponse à la flexibilité, $\Pi^{1}(f, f) \geq \Pi^{1}(i, f)$, si et seulement si :

$$
\frac{1}{9 \beta} V+\left\{\frac{1}{2} \beta\left[x-\frac{1}{3 \beta}(\mu-c)\right]^{2}-\frac{1}{6}\left[x-\frac{1}{3 \beta}(\mu-c)\right](\mu-c)\right\} \geq H
$$

La meilleure réponse résulte à nouveau de la comparaison entre le coût de la flexibilité et ses avantages. Le premier terme du membre de gauche correspond aux possibilités d'adaptation aux variations de la demande. On remarque que sa valeur est plus faible que dans le cas précédent. L'avantage informationnel offert par la flexibilité est plus grand lorsque l'autre firme est inflexible que lorsqu'elle est flexible. Le second terme du membre de gauche dépend de la capacité de la firme inflexible. Ce terme est minimum lorsque $x=\frac{1}{2 \beta}(\mu-c)$, la valeur maximale que peut prendre la capacité de l'usine inflexible sans violer les hypothèses précédentes. Il est négatif lorsque la capacité de l'usine inflexible est élevée $\left(x \geq \frac{1}{3 \beta}(\mu-c)\right)$ et positif dans le cas contraire. Ces deux fonctions de meilleure réponse permettent de caractériser les équilibres de Nash parfaits du jeu avec engagement.

Proposition 1: Les choix technologiques à l'équilibre de Nash parfait du jeu avec observation sont les suivants:

a) $(i, i)$ si les conditions (5) et (6) ne sont pas vérifiées.

b) $(f, f)$ si les conditions (5) et (6) sont vérifiées.

c) $(i, f)$ ou $(f, i)$ si la condition (5) est vérifiée alors que la condition (6) ne l'est pas.

d) $(f, f)$ et $(i, i)$ si la condition (5) n'est pas vérifiée tandis que la condition (6) l'est.

Dans les industries où l'incertitude sur le niveau de la demande est faible et où la capacité de production d'une usine inflexible est proche du niveau de production que choisiraient deux firmes se livrant une concurrence à la Cournot sans contraintes de capacité, les deux firmes choisissent des technologies inflexibles. Ces technologies permettent de produire des quantités proches des quantités souhaitées en minimisant les coûts de production. Dans les industries qui s'éloignent de ces conditions, la technologie flexible est adoptée à l'équilibre par l'une des 
firmes soit pour s'adapter aux variations d'une demande volatile, soit parce que la capacité de l'usine inflexible est trop éloignée du niveau de production optimal. Dans les industries avec une forte incertitude sur la demande ou des niveaux de production optimaux très différents de ceux d'une usine inflexible, les deux firmes choisissent la flexibilité à l'équilibre. Il existe aussi des industries caractérisées par une faible variance de la demande et un niveau de demande induisant des niveaux de production souhaités légèrement supérieurs à la capacité d'une usine inflexible. Pour ces industries, il existe deux équilibres de Nash parfaits : l'un où les deux firmes sont inflexibles, l'autre où les deux firmes sont flexibles. Dans ce genre d'industrie, la configuration technologique $(i, i)$ procure une espérance de profit plus élevée que la configuration technologique $(f, f)$. On est donc en présence de ce qu'on pourrait appeler une "trappe de flexibilité", une forme de jeu de coordination.

\subsection{Analyse du jeu sans observation}

Dans le jeu sans observation, les firmes n'observent pas le choix technologique de leur concurrente et tout se passe comme si chacune des firmes choisissait simultanément, lors de la première phase, une technologie et une fonction de décision de production pour la seconde phase dont l'argument est le niveau observé de la demande, $\alpha$. Les stratégies pertinentes sont donc les suivantes.

- Si une firme choisit $f$ et pense que son concurrent opte aussi pour $f$, elle choisit la fonction $q(\alpha)=\frac{\alpha-c}{3 \beta}$ pour la seconde étape; on note cette stratégie $S 1:\left(f, q(\alpha)=\frac{\alpha-c}{3 \beta}\right)$.

- Si une firme choisit $f$ et pense que son concurrent a choisi $i$, elle choisit $q(\alpha)=\frac{\alpha-\beta x-c}{2 \beta}$; on note cette stratégie $S 2:\left(f, q(\alpha)=\frac{\alpha-\beta x-c}{2 \beta}\right)$.

- Si une firme choisit $i$, elle ne peut produire que la quantité $q=x$, quelle que soit la technologie de l'autre firme; on note cette stratégie $S 3:(i, q=x)$.

Seules les trois stratégies précédentes peuvent apparaître à l'équilibre. Cependant pour caractériser les fonctions de meilleure réponse des firmes et définir les équilibres de Nash du jeu sans observation, il est nécessaire de calculer le gain qu'obtiendrait une firme flexible dont le concurrent aurait opté pour une technologie flexible et supposé que la première firme joue la stratégie S3. 
- Si une firme choisit $f$ et pense que son concurrent a lui aussi adopté $f$ mais qu'il conjecture qu'elle-même a choisi $i$, la firme choisit la fonction $q(\alpha)=\frac{\alpha+\beta x-c}{4 \beta}$; on note cette stratégie $S 4:\left(f, q(\alpha)=\frac{\alpha+\beta x-c}{4 \beta}\right)$.

Trois équilibres sont possibles dans le jeu sans observation : $(S 1, S 1),(S 3, S 3)$ et $(S 2, S 3)$ [ou $(S 3, S 2)]$. Dans tous les autres cas, l'une des firmes, au moins, n'est pas sur sa fonction de meilleure réponse.

La fonction de meilleure réponse $M R(\cdot)$ d'une firme aux différents choix de stratégie de sa concurrente est (voir annexe) :

- $S 3=M R(S 2)$ si et seulement si

$$
H_{1} \equiv \frac{1}{16 \beta} V+\frac{9}{16} \beta\left[x-\frac{1}{3 \beta}(\mu-c)\right]^{2} \leq H,
$$

sinon, $S 4=M R(S 2)$.

- $S 1=M R(S 1)$ si et seulement si

$$
H_{2} \equiv \frac{1}{9 \beta} V+\beta\left[x-\frac{1}{3 \beta}(\mu-c)\right]^{2} \geq H,
$$

sinon, $S 3=M R(S 1)$.

- $S 2=M R(S 3)$ si et seulement si

$$
H_{3} \equiv \frac{1}{4 \beta} V+\frac{9}{4} \beta\left[x-\frac{1}{3 \beta}(\mu-c)\right]^{2} \geq H,
$$

sinon, $S 3=M R(S 3)$.

Ces trois conditions ont une structure semblable. Il s'agit dans chacun des cas de comparer le coût fixe de la flexibilité $H$ aux deux avantages liés à la flexibilité : l'adaptation aux variations de la demande et la suppression de la restriction imposée par la capacité exogène de la technologie inflexible. On remarque en outre que : $H_{1}<H_{2}<H_{3}$.

La condition (9) est identique à la condition sous laquelle l'inflexibilité est la meilleure réponse à l'inflexibilité dans le jeu avec observation. On en déduit que la possibilité d'observer ou non le choix technologique de l'autre firme n'affecte pas cette condition et par conséquent l'équilibre $(i, i)$ émerge uniquement pour des raisons d'efficacité et non pour des raisons stratégiques. Les fonctions de meilleure réponse des firmes permettent de caractériser les équilibres du jeu sans observation. 
Proposition 2: Les équilibres de Nash parfaits du jeu sans observation sont les suivants:

a) Si $H<H_{1}$, l'équilibre est $(S 1, S 1)$ et donc les deux firmes choisissent $f$.

b) Si $H_{1} \leq H<H_{2}$, les équilibres sont $(S 2, S 3)$ et $(S 1, S 1)$, une firme choisit $f$ et l'autre $i$ ou les deux firmes choisissent $f$.

c) Si $H_{2} \leq H<H_{3}$, l'équilibre est $(S 2, S 3)$, une firme choisit $f$ et l'autre $i$.

d) $\mathrm{Si}_{3} \leq H$, l'équilibre est $(S 3, S 3)$, les deux firmes choisissent $i$.

Les équilibres des jeux avec et sans observation sont représentés sur la Figure 1 pour les valeurs suivantes des paramètres : $\beta=1, x=1, c=0,2$, et $H=0,1$ (la valeur minimale de $\underline{\alpha}$ permettant aux deux firmes d'être actives en seconde période est alors 2.2).

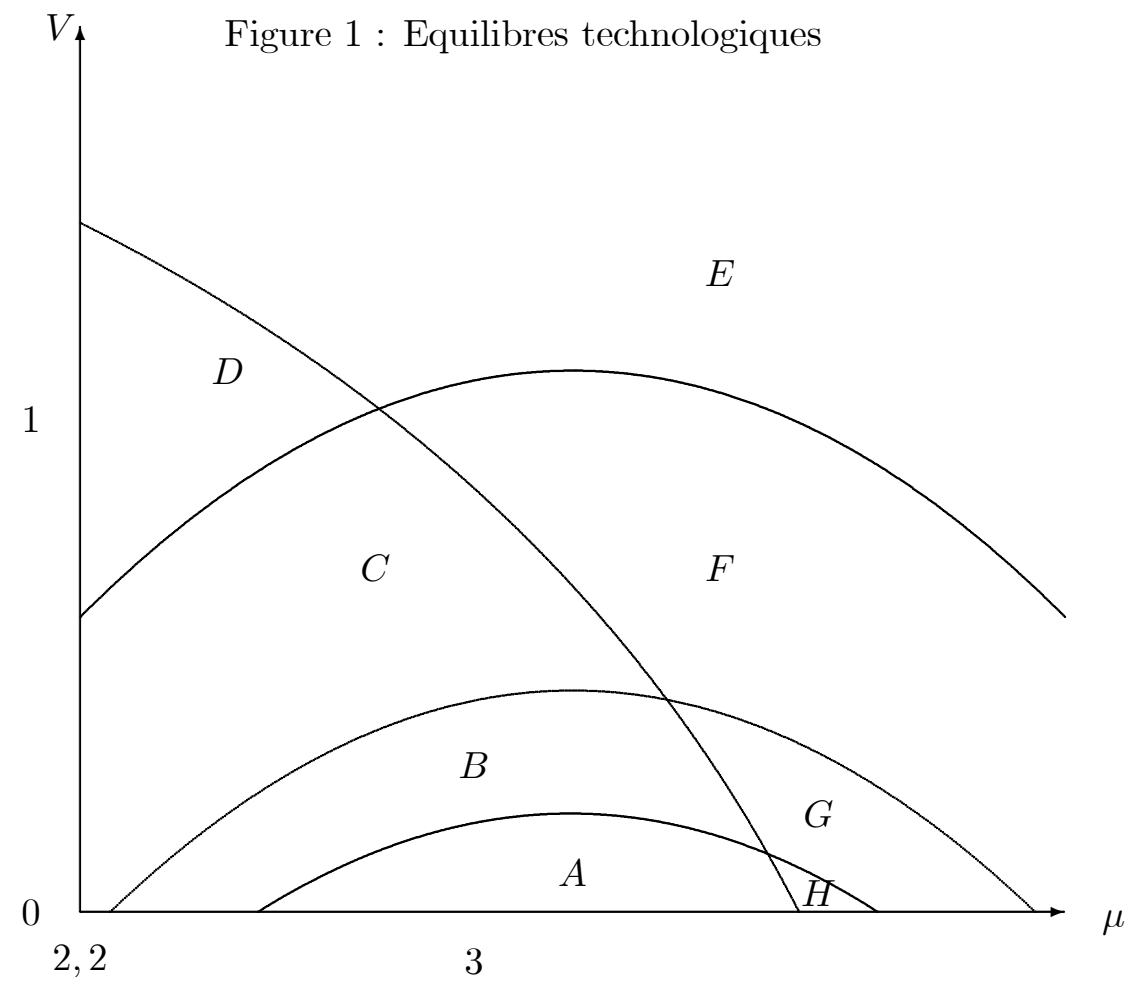

On obtient 8 régions différentes dans l'espace $(\mu, V)$ :

- Région A: l'équilibre AE est $(i, i)$; l'équilibre SO est $(i, i)$.

- Région B: l'équilibre AE est $(i, f)[(f, i)]$; l'équilibre SO est $(i, f)[(f, i)]$.

- Région C: l'équilibre AE est $(i, f)[(f, i)]$; les équilibres SO sont $(i, f)[(f, i)]$ et $(f, f)$.

- Région D: l'équilibre $\mathrm{AE}$ est $(i, f)[(f, i)]$; l'équilibre SO est $(f, f)$. 
- Région E: l'équilibre AE est $(f, f)$; l'équilibre SO est $(f, f)$.

- Région F: l'équilibre AE est $(f, f)$; les équilibres SO sont $(i, f)[(f, i)]$ et $(f, f)$.

- Région G: l'équilibre $\mathrm{AE}$ est $(f, f)$; l'équilibre the $\mathrm{SO}$ est $(i, f)[(f, i)]$.

- Région H: les équilibres $\mathrm{AE}$ sont $(i, i)$ et $(f, f)$; l'équilibre SO est $(i, i)$.

La structure des équilibres du jeu sans observation est la suivante. Si la volatilité de la demande $V$ est faible et si la taille moyenne du marché $\mu$ est proche de $3 \beta x+c\left(H_{3} \leq H\right)^{7}$, alors les deux firmes choisissent la technologie inflexible. Si l'incertitude sur le niveau de la demande, $V$, augmente ou si $\mu$ s'éloigne de la valeur $3 \beta x+c\left(H_{2} \leq H<H_{3}\right)$, alors l'une des firmes adopte la technologie flexible. Si $V$ augmente davantage ou si $\mu$ est encore plus éloignée de $3 \beta x+c$, rendant la capacité $x$ de la firme inflexible inadaptée à la taille moyenne du marché $\left(H<H_{1}\right)$, alors l'autre firme adopte, elle aussi, la technologie flexible. Toutefois, dans certaines zones $\left(H_{1} \leq H<H_{2}\right)$, le jeu $\mathrm{SO}$ admet plusieurs types d'équilibres : dans les zones $\mathrm{C}$ et $\mathrm{F}$, on a deux équilibres, $(i, f)[(f, i)]$ et $(f, f)$. Cette multiplicité des équilibres est due au fait qu'une firme flexible peut jouer deux stratégies différentes, $S 1$ et $S 2$. Or, dans ces zones, $S 1=M R(S 1)$ mais $S 3=M R(S 2)$ et $S 2=M R(S 3)$, aussi la meilleure réponse à la flexibilité dépend de la fonction de décision de production retenue par la firme flexible. L'absence d'observation des choix technologiques, avant la phase de compétition en quantités, introduit un problème de coordination entre les deux firmes.

\subsection{Comparaison des deux équilibres et valeur stratégique de la flexibilité}

La comparaison des équilibres du jeu AE à ceux du jeu SO permet de faire apparaître clairement l'influence des considérations stratégiques dans le choix technologique des firmes. Cette influence permet de mieux comprendre l'apparition d'une trappe de flexibilité dans le jeu AE.

Deux régions sont particulièrement intéressantes dans lesquelles les configurations technologiques de l'industrie qui émergent à l'équilibre des jeux AE et SO sont différentes. Dans la zone D de la Figure 1, l'équilibre du jeu AE est $(i, f)[(f, i)]$ et l'équilibre du jeu SO est $(f, f)$. Dans le jeu AE, une firme choisit l'inflexibilité pour des raisons stratégiques. Toutefois, si son concurrent ne pouvait pas observer son choix technologique, la firme choisirait une technologie flexible. Dans cette zone, la capacité $x$ d'une firme inflexible est supérieure à l'espérance

\footnotetext{
${ }^{7}$ Ce qui se produit lorsque la capacité $x$ d'une usine inflexible est proche de la quantité choisie dans un duopole à la Cournot : $\frac{1}{3 \beta}(\mu-c)$.
} 
mathématique du niveau de production d'une firme flexible. En choisissant l'inflexibilité, une firme peut par conséquent s'engager à produire plus à la seconde période du jeu, et réduire ainsi, en espérance, la production d'équilibre de l'autre firme : la valeur stratégique de la flexibilité est négative dans cette région $\mathrm{D}$. Dans la région $\mathrm{G}$, l'équilibre du jeu $\mathrm{AE}$ est $(f, f)$ tandis que l'équilibre du jeu SO est $(i, f)[(f, i)]$. Dans cette région, l'espérance mathématique du niveau de production d'une firme flexible est supérieure à la capacité $x$ d'une usine inflexible. En choisissant la flexibilité, une firme accroît, en espérance, son niveau de production de la phase 2 et par conséquent incite l'autre firme, ayant une technologie flexible, à diminuer sa production, en espérance : la valeur stratégique de la flexibilité est positive dans cette région G. Il existe deux autres zones, les zones $\mathrm{C}$ et $\mathrm{F}$, où des différences similaires peuvent être relevées mais dans ces deux zones, le jeu SO admet plusieurs équilibres, rendant la comparaison moins frappante. La flexibilité a donc une valeur stratégique positive lorsque la capacité de l'usine inflexible est faible par rapport à la taille espérée du marché et a une valeur stratégique négative lorsque cette capacité est élevée.

La caractérisation de la valeur stratégique de la flexibilité permet aussi de mieux comprendre les raisons de l'existence d'une trappe de flexibilité dans le jeu AE. Cette trappe présente dans la région $\mathrm{H}$ du jeu AE n'apparaît plus dans le jeu SO. Dans le jeu AE, si une firme est flexible alors l'autre firme choisit, elle aussi, la flexibilité : la flexibilité est la meilleure réponse à la flexibilité et $(f, f)$ est par conséquent un équilibre. Dans cette région, la production d'une firme flexible est, en espérance, plus grande que la capacité d'une usine inflexible. Aussi choisir la flexibilité quand l'autre firme est flexible diminue l'espérance de la production de l'autre firme. La valeur stratégique de la flexibilité est positive. Cependant, les firmes se trouvent dans une trappe de flexibilité parce que les deux firmes réaliseraient des profits plus importants, en espérance, dans l'autre configuration d'équilibre, $(i, i)$. Dans le jeu SO, la valeur stratégique de la flexibilité disparaît puisque les choix technologiques ne sont pas observables : la meilleure réponse à la flexibilité devient l'inflexibilité et le seul équilibre est $(i, i)$. On en déduit que l'absence de possibilité d'observation évite l'apparition d'une trappe de flexibilité. L'absence d'observation permet donc d'éliminer les problèmes d'équilibres multiples présents dans le jeu $\mathrm{AE}$ mais elle en génère d'autres qui n'existaient pas dans le jeu AE.

Il existe donc des zones de valeurs des paramètres pour lesquelles les conditions d'observation 
ont un impact sur les technologies choisies à l'équilibre. On doit s'attendre à trouver, lorsque les conditions d'observation s'améliorent, des structures technologiques plus flexibles dans les industries de tailles importantes et moins flexibles dans les industries de tailles restreintes.

Les conditions d'observation ont aussi un impact sur les sentiers d'adoption de la technologie flexible, que ce soit la taille moyenne du marché ou la volatilité de la demande qui augmente. En utilisant l'approche de Boyer et Moreaux (1997), mutatis mutandis, on trouve que la date d'adoption peut-être anticipée ou repoussée selon la région particulière considérée. Si la capacité d'une usine inflexible est grande comparée à l'espérance de la taille du marché, formellement si $\mu \leq 3 \beta x+c$, la flexibilité a une valeur stratégique négative ; par conséquent, lorsque la volatilité de la demande augmente, le passage d'une configuration technologique asymétrique à une configuration technologique symétrique et flexible $(f, f)$ a lieu plus tôt lorsque le choix de flexibilité n'est pas observable. Cela peut signifier, par exemple, que les firmes optent plus rapidement pour la flexibilité si elle est de nature organisationnelle (non observable) que si elle est de nature purement technologique (observable). $\mathrm{Si}$, au contraire, la taille d'une usine inflexible est faible comparée à l'espérance de la taille du marché, c'est-à-dire, si $\mu \geq 3 \beta x+c$, la flexibilité a une valeur stratégique positive et la transition d'une configuration technologique $(i, f)[(f, i)]$ à la configuration technologique $(f, f)$ a lieu plus tard dans le jeu SO. De façon analogue, lorsque la taille du marché augmente pour un niveau d'incertitude de la demande donné, le saut de l'équilibre $(f, f)$ à l'équilibre $(i, f)$ intervient plus tôt tandis que le passage de l'équilibre $(i, f)$ à l'équilibre $(f, f)$ est retardé dans le jeu SO.

\section{Jeu avec observation asymétrique}

Maggi (1999) a montré que le pouvoir d'engagement d'une firme dépendait de la comparaison entre l'incertitude portant sur une information privée de la firme et la possibilité d'erreur dans l'observation du message. Or, il n'y a pas de raison a priori pour que le degré d'incertitude sur l'information privée soit le même pour les deux firmes. Si l'information privée concerne le coût marginal d'une firme, celui-ci sera moins incertain pour une firme implantée depuis longtemps dans l'industrie que pour un nouvel entrant. De même, une firme peut opter pour une flexibilité technologique facilement observable alors que sa concurrente choisit une flexibilité organisationnelle en général plus difficilement détectable, d'où l'intérêt des jeux avec observation 
asymétrique.

On considère, dans cette section, le cas intermédiaire où, à la seconde phase du jeu, la firme 2 peut observer le choix technologique de la firme 1, alors que la firme 1 ne peut pas observer le choix technologique de la firme 2 .

Dans ce jeu, le concept d'équilibre pertinent n'est pas immédiat. La firme 1, n'observant pas la technologie choisie par sa rivale, est contrainte à jouer des stratégies SO. La firme 2 peut alors répondre par une meilleure réponse $\mathrm{SO}$ et donc tous les équilibres du jeu SO sont aussi des équilibres du jeu avec observation asymétrique ${ }^{8}$. De plus, le jeu avec observation asymétrique ne possède pas de sous-jeux, les équilibres SO sont donc aussi des équilibres parfaits. Mais clairement, en sélectionnant des stratégies SO, la firme 2 ne tire pas pleinement partie de l'information dont elle dispose, et il est difficile de considérer les équilibres SO comme des équilibres robustes, même s'ils sont (trivialement) parfaits. Cela impliquerait, par exemple, qu'un équilibre, dans lequel les deux firmes joueraient la stratégie S1, pourrait être soutenu par le fait que la firme 2 ne modifierait pas sa fonction de décision de production si elle observait que la firme 1 a choisi une technologie inflexible plutôt que la technologie flexible comme elle l'avait anticipé. On suppose donc que, lors de la seconde période du jeu, la firme 2 utilise une fonction de décision intégrant toute l'information disponible. Cette fonction peut, comme c'est habituellement le cas, comporter des menaces implicites non crédibles, même si la crédibilité n'est pas facile à définir dans ce jeu, qui ne comporte pas de sous-jeux, sauf dans des cas extrèmes. Un exemple de ces cas extrèmes, dans lequel la menace implicite n'est clairement pas crédible, est une fonction de production de seconde période stipulant une quantité très élevée si la firme 1 choisit une technologie flexible, dans le seul but d'obliger la firme 1 à opter pour une technologie inflexible plutôt que pour une technologie flexible. On ne peut pas considérer ces équilibres comme économiquement pertinents. Pour ces raisons, on ne considère comme robuste que les équilibres où la fonction de décision de production de la firme 2 est le résultat d'une concurrence à la Cournot étant donné les choix technologiques de la première période. Cela implique que la fonction de meilleure réponse de la firme 1, bien qu'elle joue une stratégie SO, est obtenue à partir des conditions définies dans le jeu AE. En effet, la modification de sa technologie par la firme 1 entraîne une modification de la quantité produite par la firme 2. De façon analogue,

\footnotetext{
${ }^{8}$ Les équilibres SO sont aussi des équilibres de Nash du jeu AE mais ils ne sont pas parfaits.
} 
bien que la firme 2 joue une stratégie AE, les conditions pertinentes pour calculer sa fonction de meilleure réponse sont celles du jeu SO, car la technologie de la firme 1 étant donnée, la firme 2 peut modifier son choix technologique sans que change la quantité produite par la firme 1 en seconde période.

De cet ensemble de fonctions de meilleure réponse, on déduit les équilibres du jeu avec observation asymétrique. Ces équilibres sont aussi représentés sur la Figure 1:

- Dans les régions $\mathrm{A}$ et $\mathrm{H}$, l'équilibre est $(i, i)$.

- Dans les régions $\mathrm{B}$ et $\mathrm{C}$, les équilibres sont $(i, f)$ et $(f, i)$.

- Dans la région $\mathrm{D}$, l'équilibre est $(i, f)$.

- Dans la région E, l'équilibre est $(f, f)$.

- Dans la région $\mathrm{F}$, les équilibres sont $(f, i)$ et $(f, f)$.

- Dans la région $\mathrm{G}$, l'équilibre est $(f, i)$.

Dans les régions $\mathrm{A}, \mathrm{B}$ et $\mathrm{E}$, les équilibres des jeux $\mathrm{SO}$ et $\mathrm{AE}$ sont les mêmes ; ils restent donc identiques dans le cas où l'observation est asymétrique. Dans la zone $\mathrm{C}$, les équilibres du jeu avec observation asymétrique sont identiques à ceux du jeu AE. La configuration $(f, f)$, qui est un équilibre du jeu SO, n'est pas un équilibre du jeu avec observation asymétrique. Si la firme 2 choisit la flexibilité et la firme 1 choisit l'inflexibilité, la firme 2 est conduite à diminuer sa production pour la plupart des niveaux de la demande. Dans la région $\mathrm{D}$, l'unique équilibre du jeu avec observation asymétrique est $(i, f)$. On peut alors se demander quelle est, dans cette région $\mathrm{D}$, la meilleure situation : observer ou être observé. La réponse dépend des valeurs des paramètres ; pour des niveaux faibles de la variance de la demande, la meilleure technologie est la technologie inflexible et il est préférable d'être observé que d'observer. Mais pour des niveaux élevés d'incertitude sur la demande, la meilleure technologie est la flexibilité et, par conséquent, il est préférable d'observer que d'être observé ${ }^{9}$. Dans la région F, il y a deux équilibres de Nash du jeu avec observation asymétrique, $(f, f)$ et $(f, i)$, et, dans l'équilibre $(f, i)$, il est préférable d'être flexible et donc d'être observé. Dans la région $\mathrm{G}$, l'équilibre de Nash $(f, i)$ est unique. La meilleure technologie est la technologie flexible et donc il est préférable d'être observé. Dans

\footnotetext{
${ }^{9}$ L'équation définissant la frontière entre ces deux zones est :

$$
\frac{1}{4 \beta}\left[V+(\mu-\beta x-c)^{2}\right]-\frac{1}{2} x(\mu-\beta x-c)-H=0 .
$$
}


la région $\mathrm{H}$, l'équilibre unique est $(i, i)$. Le fait que l'une des firmes ne puisse pas observer le choix technologique de sa concurrente prévient l'apparition d'une trappe de flexibilité. Cette structure intermédiaire d'observation profite donc aux deux firmes dans la région $\mathrm{H}$ tandis que dans les régions $\mathrm{D}, \mathrm{F}$ et $\mathrm{G}$, l'une des firmes gagne au détriment de l'autre.

On peut résumer les résultats précédents par le tableau suivant :

Tableau 1 : Conditions d'observation et choix technologiques d'équilibres

$\begin{array}{llll}\text { Zones } & \text { Avec engagement } & \text { Sans observation } & \text { Observation Asymétrique } \\ \text { A } & (i, i) & (i, i) & (i, i) \\ \text { B } & (i, f) \text { et }(f, i) & (i, f) \text { et }(f, i) & (i, f) \text { et }(f, i) \\ \text { C } & (i, f) \text { et }(f, i) & (i, f),(f, i) \text { et }(f, f) & (i, f) \text { et }(f, i) \\ \text { D } & (i, f) \text { et }(f, i) & (f, f) & (i, f) \\ \text { E } & (f, f) & (f, f) & (f, f) \\ \text { F } & (f, f) & (i, f),(f, i) \text { et }(f, f) & (f, i) \text { et }(f, f) \\ \text { G } & (f, f) & (i, f) \text { et }(f, i) & (f, i) \\ \text { H } & (i, i) \text { et }(f, f) & (i, i) & (i, i) \\ & (\text { trappe de flexibilité) } & & \end{array}$

\section{Observation endogène}

Dans les sections précédentes, les possibilités d'observation sont exogènes. Cette hypothèse est relativement forte. En réalité les entreprises contrôlent plus ou moins d'une part la transmission d'information vers l'extérieur et d'autre part le flux d'information qu'elles reçoivent. Toute une branche de l'économie industrielle a précisément étudié les incitations des entreprises à échanger des informations lorsque la concurrence est imparfaite. Dans ces modèles ${ }^{10}$, la demande ou le coût de production est aléatoire et chacune des firmes reçoit un signal imparfait sur la variable inconnue. Avant d'observer leurs signaux respectifs, les firmes ont la possibilité de s'engager à les mettre en commun pour disposer d'une meilleure information en créant, par exemple, une association professionnelle indépendante, chargée de collecter et de divulguer les différentes informations. Les résultats présentés par les premiers articles dépendaient fortement des hypothèses retenues. Raith (1996) a développé un modèle assez général qui permet de distinguer les différents effets en présence. Lorsque la concurrence est une concurrence à la

\footnotetext{
${ }^{10}$ Voir Raith (1996) pour les références bibliographiques.
} 
Cournot ou à la Bertrand avec une incertitude sur le niveau de la demande, la rentabilité de l'échange d'information dépend de la somme de deux effets : (1) permettre à ses concurrents d'acquérir une meilleure connaissance de leurs fonctions de profit respectives entraîne une plus grande corrélation des stratégies des firmes et l'effet sur les profits dépend de la pente des fonctions de réaction, (2) permettre à ses concurrents d'acquérir une meilleure connaissance de sa propre fonction de profit est toujours profitable.

La nature de l'information échangée dans notre modèle est assez différente, il s'agit d'un choix stratégique et pas de la valeur prise par une variable aléatoire. Cependant, la démarche proposée par les études sur le partage de l'information reste pertinente. A l'instar de Raith (1996), on considère d'abord la création d'une association professionnelle, puis un choix de divulgation non coopératif.

\subsection{Associations professionnelles}

Les firmes s'accordent pour créer une association professionnelle si l'espérance de leur profit est supérieure dans le jeu avec observation à celle obtenue dans le jeu sans observation. Dans le contexte étudié ici, cette comparaison des espérances de gains, dans les différentes situations d'observation envisagées, se heurte au problème délicat de la possible multiplicité des équilibres ${ }^{11}$. Il est donc nécessaire de choisir un critère pour sélectionner un équilibre unique parmi les différents équilibres de Nash. On suppose que les firmes se coordonnent sur l'équilibre qui procure l'espérance de la somme des profits la plus élevée. Lorsque deux équilibres asymétriques $((f, i)$ et $(i, f))$ vérifient ce critère, les firmes déterminent leur rôle respectif par un procédé aléatoire donnant la même probabilité à chacun des équilibres.

Dans les zones A, B et E, les équilibres du jeu sont les mêmes pour chacune des structures d'information envisagées. Les firmes sont donc indifférentes entre créer et ne pas créer une association professionnelle. Cependant dans la zone B, il existe deux équilibres symétriques. On n'a envisagé que des équilibres de Nash en stratégies pures où les firmes se coordonnent parfaitement ; si on avait envisagé des équilibres en stratégies mixtes entraînant des possibilités d' "erreurs", les firmes ne seraient plus indifférentes entre les différentes structures d'information. L'existence d'une association professionnelle (jeu avec observation) permettrait de limiter les

\footnotetext{
${ }^{11}$ Dans les modèles de partage de l'information, l'équilibre Bayésien du sous jeu consécutif au choix d'une structure d'information est unique.
} 
conséquences d'erreurs éventuelles lors du choix technologique.

Dans la zone $\mathrm{H}$, le jeu AE est moins favorable pour les firmes que les deux autres structures d'information car il admet comme équilibre de Nash la configuration $(f, f)$, qui est dominée par la configuration $(i, i)$ seul équilibre de Nash des deux autres jeux. Les firmes préfèrent donc ne pas créer d'association professionnelle. Cependant, comme l'équilibre $(f, f)$ est éliminé par le critère de sélection de l'équilibre défini ci-dessus, les firmes sont indifférentes entre les trois structures d'information.

Dans les autres zones, le choix de la structure d'information dépend de la comparaison des profits obtenus dans les configurations technologiques $(f, f)$ et $(f, i)$ ou $(i, f)]$.

Dans les zones $\mathrm{F}$ et $\mathrm{G}$, les configurations technologiques mixtes sont préférables à la configuration technologique $(f, f)$. En effet, dans ces zones, l'espérance de la demande est forte par rapport à la taille d'une usine inflexible. Une usine inflexible produit donc moins, en moyenne, qu'une firme flexible ce qui augmente les profits de l'industrie. L'adoption de l'inflexibilité réduit de plus les coûts fixes de l'industrie. Les configurations technologiques mixtes sont des équilibres du jeu SO tandis que le jeu $\mathrm{AE}$ admet comme seul équilibre $(f, f)$. Les firmes ont donc intérêt à s'engager à ne pas observer leurs choix technologiques respectifs. Dans ces zones, les firmes ne créent pas d'association professionnelle et jouent le jeu SO.

Dans les zones C et D, la comparaison des profits des différentes configurations technologiques dépend de la valeur des paramètres. En effet, dans ces deux zones, la taille espérée de la demande est faible par rapport à la capacité d'une usine inflexible. Une firme flexible produit donc moins, en moyenne, qu'une firme inflexible. Les profits sur coûts variables sont donc plus élevés, en moyenne, dans une industrie $(f, f)$ que dans une industrie ayant une configuration technologique mixte. Mais les coûts fixes sont plus élevés lorsque les deux firmes optent pour la flexibilité. Le premier effet l'emporte lorsque l'espérance de la demande est très faible alors que le second est dominant lorsque l'espérance de la demande est plus élevée. Dans ce dernier cas, les firmes préfèrent jouer le jeu avec observation et s'accordent donc pour créer une association professionnelle. Dans cette zone, augmenter les effets stratégiques permet aux firmes d'accroître l'espérance de leur profit ${ }^{12}$.

\footnotetext{
${ }^{12}$ Ex ante. En effet le gain est effectué par l'une des firmes au détriment de l'autre. L'accord sur la création de l'association professionnelle ne peut se faire que parce que les firmes ignorent encore quel rôle leur sera assigné.
} 


\subsection{Révélation non coopérative de l'information}

On considère maintenant que le choix des firmes de s'engager à révéler ou non leur information est effectué de façon non coopérative avant le choix technologique. Les firmes ont le choix entre deux modes d'organisation : le premier rend le choix technologique observable tandis que le second permet de conserver ce choix secret. Ce mode d'organisation peut, par exemple, être le choix d'un mode de financement : une firme peut choisir de se financer par l'émission d'actions en bourse ce qui lui donne l'obligation légale d'informer régulièrement ses actionnaires sur ses choix stratégiques ou de se financer auprès d'un nombre restreint d'investisseurs importants (banques ou sociétés de capital risque), qui ne divulgueront pas les informations qui leur seront communiquées. Il peut aussi s'agir du choix du mode de flexibilité : technologique (observable) ou organisationnelle (non observable).

Dans les zones A, B, E et $\mathrm{H}$, choisir de révéler ou non l'information n'influence pas le déroulement de la suite du jeu. Les modes d'organisation choisis restent donc indéterminés.

Dans la zone G, la structure d'observation qui maximise le profit joint des firmes est celle du jeu SO. Mais les firmes ont intérêt à dévier de cette situation en choisissant de s'engager à divulguer leur information. En effet, si l'une des firmes dévie, le jeu consécutif est le jeu avec observation asymétrique ; or, comme on l'a noté à la section 4, dans cette zone il est préférable d'être observé que d'observer. En déviant, une firme peut sélectionner la configuration technologique qui lui est la plus favorable. L'autre firme se retrouve alors dans la situation la plus défavorable pour elle; elle choisit donc aussi de rendre son choix technologique observable. C'est donc la configuration AE qui émerge à l'équilibre. L'étape du choix de divulgation de l'information a dans cette zone une structure de jeu du dilemme du prisonnier. La zone F présente les mêmes caractéristiques que la zone $\mathrm{G}$, à la différence près qu'il existe d'autres équilibres de Nash mais ceux-ci sont éliminés par le critère de raffinement adopté précédemment.

Dans la zone C, les configurations technologiques mixtes sont des équilibres de Nash dans les trois structures d'information. Le jeu sans observation admet en plus la configuration $(f, f)$ comme équilibre de Nash. Lorsque les valeurs des paramètres sont telles que cette dernière configuration est celle qui maximise les profits joints des deux firmes, alors la structure d'information SO est celle qui émerge à l'équilibre. Dans le cas contraire, le critère de raffine- 
ment rend le déroulement du jeu indépendant de la structure d'information choisie, celle-ci est donc indéterminée.

Dans la zone D, le meilleur rôle dépend de la valeur des paramètres dans le jeu avec observation asymétrique. Pour des niveaux faibles de la demande, il est préférable d'être observé que d'observer. La stratégie dominante des firmes est alors de s'engager à révéler son choix technologique et la structure AE apparaît à l'équilibre. Pour les niveaux les plus faibles de la demande, la structure SO est celle qui maximise le profit joint des firmes, celles-ci sont donc dans un dilemme du prisonnier. Pour des niveaux élevés d'incertitude, il est préférable d'observer que d'être observé. La stratégie dominante des firmes est alors de s'engager à ne pas révéler leur choix technologique. La structure d'équilibre est donc SO. Les firmes seraient dans une situation préférable si elles avaient pû mettre en place une structure d'observation AE. Dans cette zone les firmes sont de nouveau confrontées à un dilemme du prisonnier.

Lorsque l'information est révélée de manière non coopérative, les structures d'observation qui émergent sont, dans les zones où elles ont un impact, diamétralement opposées à celles qui émergent lorsque ce choix est fait de manière coopérative. On remarque, en outre, que, dans les zones où l'observabilité à un impact, la structure d'observation asymétrique n'est jamais un équilibre de Nash.

\section{Conclusion}

On a montré que la possibilité d'observer ou non les choix technologiques de ses concurrents a un impact sensible sur les choix technologiques et sur les dates d'adoption des technologies flexibles lorsque le marché s'accroît ou lorsque l'incertitude sur le niveau de la demande augmente. On a précisé la signification de la valeur stratégique de la flexibilité et montré qu'elle pouvait être positive ou négative selon les fondamentaux de l'industrie.

Dans les marchés de tailles importantes, la valeur stratégique de la flexibilité est positive et donc l'observation des choix technologiques favorise l'adoption de technologies flexibles. Dans les marchés de tailles plus faibles, la valeur stratégique de la flexibilité est négative et l'observation des choix amène les firmes à choisir des technologies moins flexibles. Lorsque les firmes peuvent influencer la structure d'observation du jeu, elles choisissent, lorsque le marché est de taille importante, de ne pas observer leurs décisions technologiques réciproques lorsque ce choix est 
effectué de manière coopérative mais elles optent pour l'observation lorsque ce choix est non coopératif. Lorsque le marché est de taille restreinte, la structure d'observation qui émerge à l'équilibre dépend de la volatilité de la demande. 


\section{APPENDICE: Caractérisation de la meilleure réponse dans le jeu SO.}

1. Meilleure Reponse a $S 1:\left(f, q(\alpha)=\frac{\alpha-c}{3 \beta}\right)$.

Si la firme 2 joue $S 1$, alors la firme 1 peut choisir d'être flexible en jouant la stratégie $S 1$ ou d'être inflexible en jouant la stratégie $S 3$. Si la firme 1 joue $S 3$, alors

$$
q_{1}=x, q_{2}=\frac{\alpha-c}{3 \beta}, p=\frac{2 \alpha-3 x \beta+c}{3}, \pi_{1}=x \frac{2 \alpha-3 x \beta-2 c}{3}, E\left(\pi_{1}\right)=x \frac{2 \mu-3 x \beta-2 c}{3}
$$

Étant donné (1), $S 1=M R(S 1)$ si et seulement si

$$
\frac{1}{9 \beta}\left[V+(\mu-c)^{2}\right]-H \geq x \frac{2 \mu-3 x \beta-2 c}{3}
$$

et donc si et seulement si

$$
\frac{1}{9 \beta} V+\beta\left[x-\frac{1}{3 \beta}(\mu-c)\right]^{2} \geq H
$$

2. Meilleure Reponse a $S 2:\left(f, q(\alpha)=\frac{\alpha-x \beta-c}{2 \beta}\right)$.

Si la firme 2 joue $S 2$, alors la firme 1 peut choisir une technologie inflexible en jouant la stratégie $S 3$ ou une technologie flexible en jouant la stratégie $S 4$. Si la firme 1 joue $S 4$, alors

$$
p=\frac{1}{4}(\alpha+x \beta+3 c), \pi_{1}=\frac{1}{16 \beta}(\alpha+x \beta-c)^{2}-H, E\left(\pi_{1}\right)=\frac{1}{16 \beta}\left[V+(\mu+x \beta-c)^{2}\right]-H
$$

Étant donné (2), $S 3=M R(S 2)$ si et seulement si

$$
\frac{1}{2} x(\mu-\beta x-c) \geq \frac{1}{16 \beta}\left[V+(\mu+x \beta-c)^{2}\right]-H
$$

et donc si et seulement si

$$
\frac{1}{16 \beta} V+\frac{9}{16} \beta\left[x-\frac{1}{3 \beta}(\mu-c)\right]^{2} \leq H
$$

3. Meilleure Reponse a $S 3:(i, q=x)$.

Si la firme 2 joue $S 3$, alors la firme 1 peut opter pour la flexibilité en jouant la stratégie $S 2$ ou être inflexible en jouant la stratégie $S 3$. Étant donnés (3) et (4), on obtient $S 2=M R(S 3)$ si et seulement si

$$
\frac{1}{4 \beta}\left[V+(\mu-x \beta-c)^{2}\right]-H \geq x(\mu-2 \beta x-c)
$$

et donc si et seulement si

$$
\frac{1}{4 \beta}\left[V+(\mu-3 x \beta-c)^{2}\right] \geq H
$$




\section{References}

[1] BAGWELL K. (1995), Commitment and observability in games, Games and Economic Behavior, 8, 271-280.

[2] BOYER M. et M. MOREAUX (1997), Capacity Commitment versus Flexibility, The Journal of Economics and Management Strategy, 6, 347-376.

[3] BOYER M., A. JACQUES et M. MOREAUX (2001a), Bankruptcy cost, financial structure and technological flexibility choices, mimeo, GREMAQ (Toulouse) et CIRANO (Montréal).

[4] BOYER M., A. JACQUES et M. MOREAUX (2001b), Observability and Product Flexibility in Oligopoly, mimeo, GREMAQ (Toulouse) et CIRANO (Montréal).

[5] FUDENBERG D. et J. TIROLE (1984), The fat-cat effect, the puppy-dog ploy and the lean and hungry look, American Economic Review, Papers and proceedings, 74, 361-366.

[6] FUDENBERG D. et J. TIROLE (1991), Game Theory, MIT Press.

[7] JACQUES A. (2000), La flexibilité technologique : un survol de la littérature, chapitre 1 de L'adoption des technologies flexibles et ses implications concurrentielles, thèse, Université des sciences sociales de Toulouse.

[8] KIM T., L-H. RÖLLER et M. TOMBAK (1994), On the timing of adoption of multiproduct technologies, Naval Research Logistics, 41, 377-394.

[9] MAGGI G. (1999), The value of commitment with imperfect observability and private information, RAND Journal of economics, 30, 555-574.

[10] MILGROM P. et J. ROBERTS (1990), The economics of modern manufacturing : technology, strategy and organization, American Economic Review, 80, 511-528.

[11] RAITH M. (1996), A general model of information sharing in oligopoly, Journal of Economic Theory, 71, 260-288.

[12] RÖLLER L-H. et M. TOMBAK (1990), Strategic choice of flexible production technologies and welfare implications, Journal of Industrial Economics, 38, 417-431. 
[13] SANCHEZ R. (1995), Strategic flexibility in product competition, Strategic Management Journal, 16, 135-159.

[14] STIGLER G. (1939), Production and distribution in the short run, Journal of Political Economy, 47, 305-327.

[15] VIVES X. (1989), Technological Competition, Uncertainty, and Oligopoly, Journal of Economic Theory, 48, 386-415. 


\section{Liste des publications au CIRANO*}

Série Scientifique / Scientific Series (ISSN 1198-8177)

2002s-12 Observation, Flexibilité et Structures Technologiques des Industries / Marcel Boyer, Armel Jacques et Michel Moreaux

2002s-11 Idiosyncratic Consumption Risk and the Cross-Section of Asset Returns / Kris Jacobs et Kevin Q. Wang

2002s-10 The Demand for the Arts / Louis Lévy-Garboua et Claude Montmarquette

2002s-09 Relative Wealth, Status Seeking, and Catching Up / Ngo Van Long, Koji Shimomura

2002s-08 The Rate of Risk Aversion May Be Lower Than You Think / Kris Jacobs

2002s-07 A Structural Analysis of the Correlated Random Coefficient Wage Regression Model / Christian Belzil et Jörgen Hansen

2002s-06 Information Asymmetry, Insurance, and the Decision to Hospitalize / Åke Blomqvist et Pierre Thomas Léger

2002s-05 Coping with Stressful Decisions: Individual Differences, Appraisals and Choice / Ann-Renée Blais

2002s-04 A New Proof Of The Maximum Principle / Ngo Van Long et Koji Shimomura

2002s-03 Macro Surprises And Short-Term Behaviour In Bond Futures / Eugene Durenard et David Veredas

2002s-02 Financial Asset Returns, Market Timing, and Volatility Dynamics / Peter F. Christoffersen et Francis X. Diebold

2002s-01 An Empirical Analysis of Water Supply Contracts / Serge Garcia et Alban Thomas

2001s-71 A Theoretical Comparison Between Integrated and Realized Volatilities Modeling / Nour Meddahi

2001s-70 An Eigenfunction Approach for Volatility Modeling / Nour Meddahi

2001s-69 Dynamic Prevention in Short Term Insurance Contracts / M. Martin Boyer et Karine Gobert

2001s-68 Serial Cost Sharing in Multidimensional Contexts / Cyril Téjédo et Michel Truchon

2001s-67 Learning from Strike / Fabienne Tournadre et Marie-Claire Villeval

2001s-66 Incentives in Common Agency / Bernard Sinclair-Desgagné

2001s-65 Detecting Mutiple Breaks in Financial Market Volatility Dynamics / Elena Andreou et Eric Ghysels

2001s-64 Real Options, Preemption, and the Dynamics of Industry Investments / Marcel Boyer, Pierre Lasserre, Thomas Mariotti et Michel Moreaux

2001s-63 Dropout, School Performance and Working while in School: An Econometric Model with Heterogeneous Groups / Marcel Dagenais, Claude Montmarquette et Nathalie Viennot-Briot

* Consultez la liste complète des publications du CIRANO et les publications elles-mêmes sur notre site Internet : 
2001s-62 Derivatives Do Affect Mutual Funds Returns : How and When? / Charles Cao, Eric Ghysels et Frank Hatheway

2001s-61 Conditional Quantiles of Volatility in Equity Index and Foreign Exchange Data / John W. Galbraith, Serguei Zernov and Victoria Zinde-Walsh

2001s-60 The Public-Private Sector Risk-Sharing in the French Insurance "Cat. Nat. System" / Nathalie de Marcellis-Warin et Erwann Michel-Kerjan

2001s-59 Compensation and Auditing with Correlated Information / M. Martin Boyer et Patrick González

2001s-58 Resistance is Futile: An Essay in Crime and Commitment / M. Martin Boyer

2001s-57 The Unreliability of Output Gap Estimates in Real Time / Athanasios Orphanides et Simon van Norden

2001s-56 Exact Nonparametric Two-Sample Homogeneity Tests for Possibly Discrete Distributions / Jean-Marie Dufour et Abdeljelil Farhat

2001s-55 Les coûts de la réglementation : une revue de la littérature / Robert Gagné, Paul Lanoie, Pierre-Carl Micheud et Michel Patry

2001s-54 Testing for structural Change in the Presence of Auxiliary Models / Eric Ghysels et Alain Guay

2001s-53 Environmental Regulation and Productivity: New Findings on the Porter Hypothesis / Paul Lanoie, Michel Patry et Richard Lajeunesse

2001s-52 The Aftermarket Performance of Initial Public Offerings in Canada / Maher Kooli et Jean-Marc Suret

2001s-51 Capital Structure and Risk Management / Karine Gobert

2001s-50 The Underpricing of Initial Public Offerings: Futher Canadian Evidence / Maher Kooli et Jean-Marc Suret

2001s-49 How Innovative Are Canadian Firms Compared to Some European Firms? A Comparative Look at Innovation Surveys / Pierre Mohnen et Pierre Therrien

2001s-48 A Tale of Two Ports / Ngo Van Long et Kar-yiu Wong

2001s-47 Wage Policy of Firms: An Empirical Investigation / Stéphanie Lluis

2001s-46 Forecasting Some Low-Predictability Time Series Using Diffusion Indices / Marc Brisson, Bryan Campbell et John W. Galbraith

2001s-45 The Importance of the Loss Function in Option Pricing / Peter Christoffersen et Kris Jacobs

2001s-44 Let's Get "Real" about Using Economic Data / Peter Christoffersen, Eric Ghysels et Norman R. Swanson

2001s-43 Fragmentation, Outsourcing and the Service Sector / Ngo Van Long, Ray Riezman et Antoine Soubeyran

2001s-42 Nonlinear Features of Realized FX Volatility / John M. Maheu et Thomas H. McCurdy

2001s-41 Job Satisfaction and Quits: Theory and Evidence from the German Socioeconomic Panel / Louis Lévy-Garboua, Claude Montmarquette et Véronique Simonnet 